\title{
Comparative efficacy of admission cardiotocography in high risk and low risk pregnancies in predicting neonatal outcome
}

\author{
Gurpreet Kaur Nandmer*, Shivangi Jaiswal, Pooja Namdeo
}

\begin{abstract}
Department of Obstetrics and Gynecology, Peoples College of Medical Science and Research Centre, Bhopal, Madhya Pradesh, India
\end{abstract}

Received: 20 August 2021

Revised: 10 September 2021

Accepted: 13 September 2021

\author{
*Correspondence: \\ Dr. Gurpreet Kaur Nandmer, \\ E-mail: kaurgauri2@yahoo.com
}

Copyright: () the author(s), publisher and licensee Medip Academy. This is an open-access article distributed under the terms of the Creative Commons Attribution Non-Commercial License, which permits unrestricted non-commercial use, distribution, and reproduction in any medium, provided the original work is properly cited.

\begin{abstract}
Background: Admission cardiotocography (CTG) is a test of fetal wellbeing performed during labor. A normal CTG is for 20-30 minutes and ranges from 110-160 bpm with beat to beat variability, two accelerations and no decelerations with two contractions (as per NICE 2018 guidelines). To study comparative efficacy of admission CTG in predicting neonatal out in high risk, low risk and universally in all pregnancy.

Methods: All high risk as well as low risk patients coming to labor room in labor were included in the study. CTG print was taken, and neonatal outcome as NICU admission were noted after taking written consent.

Results: 189 cases were taken.138 were high risk cases, CTG was taken: 91were reassuring, 47were abnormal (nonreassuring +abnormal). In terms of NICU admission, 21 and 26 neonates were admitted. Out of 51 low risk cases, 33 had reassuring CTG, and18 had abnormal; 7and 6 was the number of NICU admission, respectively. When compared, highest sensitivity, specificity and positive predictive value of $55.31 \%, 77 \%$ and $55.3 \%$ was seen in high risk pregnancy, whereas negative predictive value $(78.8 \%$ ) was slightly higher in low risk cases.

Conclusions: Admission CTG is a good predictor of neonatal outcome. Its predictive efficacy is more in high risk cases. It's practicality in revealing poor neonatal outcome is more.
\end{abstract}

Keywords: Admission CTG, High risk pregnancy, Neonatal outcome

\section{INTRODUCTION}

Admission cardiotocography (CTG) or admission test (AT) or labor admission test (LAT) is a test of fetal health that is performed when a lady is admitted in labour. It comprises a cardiotocographic tracing of 20-30 minutes done on admission. ${ }^{1}$ A normal trace shows a within range fetal heart rate with beat to beat variability, two accelerations and no decelerations with two contractions in 20 minutes (the criteria are predefined and established; as per NICE 2017 guidelines). It was introduced as a screening test in early labour to detect compromised fetuses i.e. fetuses that may be hypoxic already, or may not withstand the stress of uterine contractions. Compromised fetuses may then be delivered or subjected to additional tests of fetal surveillance like continuous CTG (cardiotocography) throughout labor in order to prevent adverse outcomes. Even a foetus which is apparently normal in the antenatal period may develop distress during labour, hence the importance of this test. Several studies including RCT support its role in high risk pregnancy. Neilson et al have recommended that electronic fetal monitoring should be used in uncomplicated labour. ${ }^{2}$ The aim of our study was to determine comparative efficacy of admission CTG in predicting neonatal out in high risk, low risk and universally in all pregnancy.

\section{METHODS}

A prospective observational study conducted in People's Hospital and Research Centre, Bhopal, India. Study period was between December 2018 and September 2019, after 
institutional ethical approval. Study population being all pregnant women (high risk as well as low risk)- at term getting admitted in the labour ward in early or established labour. Informed and written consent was obtained from the mothers enrolled in the study.
Clinical details (history and examination findings) were noted on study proforma and the admission CTG (as defined in introduction) was done for 20 minutes. $^{3}$

Table 1: CTG findings.

\begin{tabular}{|c|c|c|c|c|}
\hline & Baseline (bpm) & Variability (bpm) & Decelerations & Accelerations \\
\hline Reassuring & $110-160$ & $\geq 5$ & None & Present \\
\hline \multirow{2}{*}{$\begin{array}{l}\text { Non- } \\
\text { reassuring }\end{array}$} & 100-109 & \multirow{2}{*}{$\begin{array}{l}<5 \text { for } 40-90 \\
\text { minutes }\end{array}$} & \multirow{2}{*}{$\begin{array}{l}\text { Typical variable decelerations } \\
\text { over } 90 \text { min. or single prolonged } \\
\text { deceleration for up to } 3 \text { minutes }\end{array}$} & \multirow{2}{*}{$\begin{array}{l}\text { The absence of accelerations } \\
\text { with otherwise normal trace is } \\
\text { of uncertain significance. }\end{array}$} \\
\hline & $161-180$ & & & \\
\hline \multirow{3}{*}{ Abnormal } & $<100$ & \multirow{3}{*}{$\begin{array}{l}<5 \text { for }>90 \\
\text { minutes }\end{array}$} & \multirow{3}{*}{$\begin{array}{l}\text { Persistent atypical variable } \\
\text { decelerations or late decelerations } \\
\text { or single prolonged deceleration } \\
\text { for }>3 \text { minutes }\end{array}$} & \\
\hline & $>180$ & & & \\
\hline & $\begin{array}{l}\text { Sinusoidal pattern } \\
>10 \text { minutes }\end{array}$ & & & \\
\hline
\end{tabular}

Based on the CTG finding, further management of the woman in labour was done as per the standard departmental protocols.

The neonatal outcomes were noted in terms of admission to NICU.

\section{Inclusion criteria}

All term patients (37 weeks-42 weeks) and singleton pregnancy.

\section{Exclusion criteria}

Anomalous baby, preterm labor, IUFD, multifoetal pregnancy, malpresentations.

\section{Statistical analysis}

Statistical testing was conducted with the statistical package for the social science system version SPSS 17.0. Continuous variables were presented as mean (SD) or median if the data was unevenly distributed. Categorical variables were expressed as frequencies and percentages. Nominal categorical data between the groups was compared using Chi-squared test or Fisher's exact test as appropriate. Non-normal distribution continuous variables were compared using Wilcoxon signed rank test. For all statistical tests, a p value less than 0.05 was taken to indicate a significant difference.

\section{RESULTS}

The present study included a total of 189 women who presented in labor room of Department of Obstetrics and Gynaecology, PCMS and RC, Bhopal during study period. Out of all 189 cases, $138(73 \%)$ were high risk cases and $51(27 \%)$ were low risk cases. Admission CTG was done (Table 2).
Table 2: Cases enrolled.

\begin{tabular}{|ll|}
\hline Cases & Total $(\mathbf{n = 1 8 9})$ \\
\hline High risk & $138(73 \%)$ \\
\hline Low risk & $51(27 \%)$ \\
\hline
\end{tabular}

Of all 138 high risk cases, 91 CTG were reassuring comprising $79.8 \%$ of all reassuring CTGs, and CTGs were abnormal (non-reassuring + abnormal) comprising $72.3 \%$ of all. Out of 51 low risk cases, $33(20.2 \%)$ had reassuring CTG and 18 (37.7\%) had abnormal CTG (Table 3).

Table 3: Correlation of high risk/low risk and CTG.

\begin{tabular}{|lll|}
\hline CTG & Reassuring & Abnormal \\
\hline High risk & $91(79.8 \%)$ & $47(72.3 \%)$ \\
\hline Low risk & $33(20.2 \%)$ & $18(37.7 \%)$ \\
\hline Total & $124(100 \%)$ & $65(100 \%)$ \\
\hline
\end{tabular}

Table 4: Correlation between CTG and admission to NICU in high risk cases.

\begin{tabular}{|c|c|c|c|c|}
\hline \multirow{2}{*}{ CTG } & \multicolumn{2}{|c|}{ Admission to NICU } & \multirow{2}{*}{$\begin{array}{l}\text { Total } \\
(n=189)\end{array}$} & \multirow{2}{*}{$\begin{array}{l}P \\
\text { value }\end{array}$} \\
\hline & No & Yes & & \\
\hline Reassuring & $\begin{array}{l}70 \\
(79.7 \%)\end{array}$ & $\begin{array}{l}21 \\
(23 \%)\end{array}$ & $\begin{array}{l}91 \\
(100 \%)\end{array}$ & \multirow[b]{2}{*}{$<0.001$} \\
\hline Abnormal & $\begin{array}{l}21 \\
(45.7 \%)\end{array}$ & $\begin{array}{l}26 \\
(55.3 \%)\end{array}$ & $\begin{array}{l}47 \\
(100 \%)\end{array}$ & \\
\hline Total & 91 & 47 & 138 & \\
\hline
\end{tabular}

When we see correlate results obtained on CTG with NICU admission rate in newborns, it was seen that out of 91 reassuring CTGs, 21 (23\%) neonates were admitted in NICU. Out of 47 abnormal CTGs, 26 (55.3\%) neonates were admitted in NICU. In high risk cases, $\mathrm{p}$ value of $<0.001(\mathrm{CI}=95 \%, \mathrm{n}=138)$ was calculated, showing a significant role of CTG in predicting neonatal outcome in high risk cases (Table 4). 


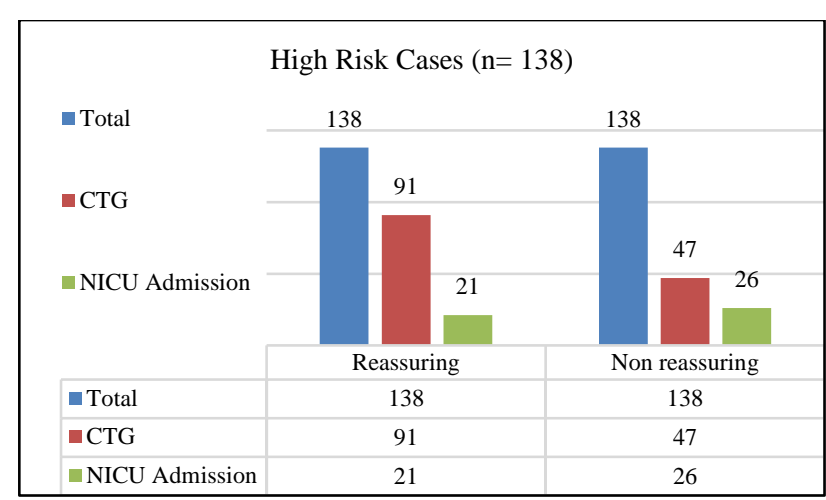

Figure 1: Relation between CTG finding in high risk cases and NICU admission.

Table 5: Correlation of admission CTG and admission to NICU in low risk cases.

\begin{tabular}{|c|c|c|c|c|}
\hline \multirow[t]{2}{*}{ CTG } & \multicolumn{2}{|c|}{$\begin{array}{l}\text { Admission to } \\
\text { NICU }\end{array}$} & \multirow{2}{*}{$\begin{array}{l}\text { Total } \\
(n=81)\end{array}$} & \multirow{2}{*}{$\begin{array}{l}P \\
\text { value }\end{array}$} \\
\hline & No & Yes & & \\
\hline Reassuring & $\begin{array}{l}26 \\
(79 \%)\end{array}$ & $\begin{array}{l}7 \\
(21 \%)\end{array}$ & $\begin{array}{l}33 \\
(100 \%)\end{array}$ & \multirow{3}{*}{0.772} \\
\hline Abnormal & $\begin{array}{l}12 \\
(67 \%)\end{array}$ & $\begin{array}{l}6 \\
(33 \%)\end{array}$ & $\begin{array}{l}18 \\
(100.0 \%)\end{array}$ & \\
\hline Total & 38 & 13 & 51 & \\
\hline
\end{tabular}

On comparing CTG results with NICU admission in low risk cases, following observations were made: out of 33 reassuring CTGs, $7(21 \%)$ neonates were admitted in NICU. Out of 18 abnormal CTGs, $6(33 \%)$ neonates were admitted in NICU. P value of $>0.05$ was found in low risk cases, and thus there was no significance and usefulness. (Table 5). Figure 2 shows the same.

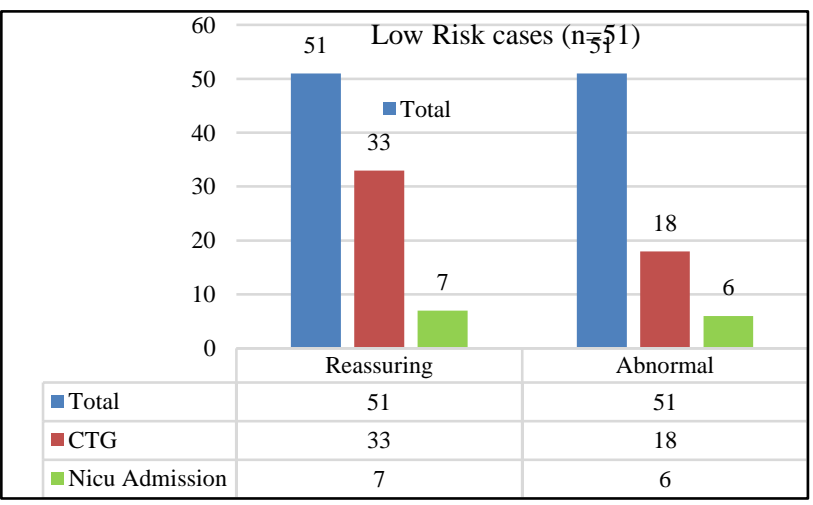

Figure 2: Relation between CTG finding in low risk cases and NICU admission.

Sensitivity, specificity, positive predictive value (PPV) and negative predictive value (NPV) of CTG in predicting neonatal outcome were calculated (Table 6). On comparing efficacy of admission CTG in high risk cases and low risk cases the sensitivity $(55.3 \%$ versus $46.15 \%)$, specificity (77 \% versus $68.4 \%)$, PPV (55.3\% versus $33.3 \%)$ and NPV (77\% versus $78 \%)$ all being highest in high risk cases as compared to low risk and universal, respectively (Table 6).

Table 6: Comparative efficacy of CTG in high risk cases and low risk cases.

\begin{tabular}{|lll|}
\hline & $\begin{array}{l}\text { High risk } \\
\text { cases }(\mathbf{n = 1 3 8})\end{array}$ & $\begin{array}{l}\text { Low risk cases } \\
(\mathbf{n = 5 1})\end{array}$ \\
\hline Sensitivity & $55.3 \%$ & $46.15 \%$ \\
\hline Specificity & $77 \%$ & $68.4 \%$ \\
\hline $\begin{array}{l}\text { Positive predictive } \\
\text { value (PPV) }\end{array}$ & $55.3 \%$ & $33.3 \%$ \\
\hline $\begin{array}{l}\text { Negative predictive } \\
\text { value (NPV) }\end{array}$ & $77 \%$ & $78.8 \%$ \\
\hline
\end{tabular}

\section{DISCUSSION}

Admission CTG is a good predictor of neonatal outcome. In the recent years, CTG has emerged as the most reliable, trustable, and non-invasive method of intrapartum foetal monitoring among different modalities available for foetal surveillance. It has become a routine in developed as well as developing countries to perform Admission CTG in all women presenting in labor including both high risk and low group.

CTG traces were divided into reassuring, and abnormal as per NICE Criteria 2017. In present study, 124 (65.6\%) women had reassuring CTG, 65 (34.4\%) had abnormal pattern.

In a study conducted on mixed population Mires et al reported $21.8 \%$ of the admission traces to be normal, while $3.6 \%$ were considered as abnormal. ${ }^{4}$ Rajalekshmi et al studied on 400 women, out of which 267 (66.75\%) had reactive tracing, $133(33.25 \%)$ had abnormal tracings, respectively. ${ }^{5}$ In study by Dhanalakshmi et al out of $481 / 800(60.1 \%)$ of women had reassuring CTG, $33.4 \%$ belonged to non-reactive group and $6.5 \%$ belonged to ominous. $^{6}$ In a study conducted in Kandan Karibu Hospital, Singapore (1998) on 1000 antenatal women, tracings were reactive in $982(4.3 \%)$, suspicious in 49 (4.7\%), and ominous $10(1 \%)$.

In present study we included both high risk and low group. Majority i.e., 138 out of 189 (73\%) women belong to high risk group. Out of these, 91 (65.9\%) had reassuring CTG, and $47(34 \%)$ had abnormal trace. In our study, $72.3 \%$ of total abnormal CTG were present in high risk group.

In study by Bhartiya et al, 72 (36\%) were low-risk and 128 (64\%) were high-risk women. ${ }^{7}$ Rajalekshmi et al in their study had 267/400 (66.755) low risk cases whereas 133 (33.25\%) high risk case. ${ }^{5}$ In a study conducted on 201 high risk cases by Gupta et al, similar results as ours was found $63.2 \%$ had reactive CTG pattern and $36.8 \%$ had nonreactive CTG pattern. ${ }^{8}$ In study by Dhakre et al, reassuring, non-reassuring and abnormal CTG pattern was observed in $62.9 \%, 13.3 \%$ and $23.8 \%$ of high risk pregnancies 
respectively. ${ }^{9}$ Manning et al, in their study done on 150 high risk cases found $83 \%$ reactive CTGs, and $17 \%$ nonreactive CTG. ${ }^{10}$

AT was a useful screening tool in patients with high risk factors and was able to predict neonatal outcomes in terms of NICU admission and the need for caesarean birth in study by Akhavan et al. ${ }^{11}$

In the present study, $44.6 \%$ neonates with abnormal trace had NICU admission. In the high risk versus low risk group, NICU admission was present in $55.3 \%$ versus $46.15 \%$, and $44.6 \%$ versus $53.8 \%$ of neonates with in abnormal and reassuring, respectively.

Rahman et al had 57.1\% NICU admission in cases with abnormal CTG. Thus, CTG prove to be a good predictor of neonatal outcome in terms of NICU admission in high risk group. ${ }^{12}$

In present study, sensitivity of admission CTG was $55.3 \%$, specificity was $77 \%$, PPV was $55.3 \%$ and NPV was found to be $77 \%$ in high risk cases whereas it's $46.1 \%, 68.4 \%$, $33.3 \%$, and $78.8 \%$, respectively in low risk cases. All criteria except NPV, were higher in high risk cases implicating CTG being a better predictor in high risk cases. High specificity and NPV in our study means that it high predictability in cases who had adverse outcome, and rules out who did not have adverse outcome.

Kushtagi et al also found low sensitivity and PPV of 53\% and $61 \%$, and high specificity and NPV of $93 \%$ and $91 \%$, respectively. ${ }^{13}$ Ducey et al got best PPV $(75 \%)$ in their results, sensitivity and specificity was $57 \%$ and $98 \%$, respectively. ${ }^{14}$

Ingemarsson et al showed got best specificity (99\%) in their results, sensitivity was $23.5 \%$, and PPV was $40 .{ }^{15}$ In most of the studies including ours, sensitivity was low indicating low number of adverse results predicted correctly by admission CTG and also, PPV was low suggestive of only few of those predicted positive were actually positive.

The few limitations of our study are that sample size taken was small. Also, no further test of foetal well-being like fetal blood sampling or fetal scalp blood $\mathrm{pH}$ was done.

\section{CONCLUSION}

CTG is a good predictor of newborn outcome. P value of $>0.05$ was found in low risk cases, and thus there was no significant usefulness of CTG in low risk cases.

$\mathrm{P}$ value of $<0.001$ was seen in high risk cases, showing a highly significant role of CTG in predicting neonatal outcome in high risk cases. Therefore, it's practicality in revealing poor neonatal outcome is more in high risk cases with sensitivity, specificity, and positive predictive value (PPV) being greater, and $\mathrm{p}$ value of $<0.001$. Thus, it should be used in high risk cases rather than using universally in all patients presenting in a labor ward.

\section{Funding: No funding sources}

Conflict of interest: None declared

Ethical approval: The study was approved by the Institutional Ethics Committee

\section{REFERENCES}

1. Behuria S, Nayak R. Admission cardiotocography as a screening test in high risk pregnancies and its corelation with peri-natal outcome. Int $\mathrm{J}$ Reprod Contracept Obstet Gynecol. 2016;5(10):3525-8.

2. Neilson JP. Fetal electrocardiogram (ECG) for fetal monitoring during labour. Cochrane Database Syst Rev. 2006;19(3):CD000116.

3. Nice clinical guideline 55. Intrapartum care: care of healthy women and their babies during childbirth. National institute for health and clinical excellence. London: RCOG Press; 2007.

4. Mires G, Williams F, Howie P. Randomised controlled trial of cardiotocography versus Doppler auscultation of the fetal heart at admission in labour in low risk obstetric population. BMJ. 2001;322:145762.

5. Rajalekshmi M, Chithra J, Nithya R, Vijay $N$. Admission cardiotocography as a screening test to predict foetal outcome and mode of delivery. Indian $\mathbf{J}$ Obstet Gynecol Res. 2016;3:43-50.

6. Dhanalakshmi K. Validity of admission test as a screening test in differentiating high and low observational antenatal women. Madras Medical College, Chennai. 2009.

7. Bhartiya V, Sharma R, Kumar A, Srivastava H. Admission cardiotocography: a predictor of neonatal outcome. J Obstet Gynecol India. 2016;66(1):321-9.

8. Gupta M, Gupta P. Role of cardiotocography in high risk pregnancy and its correlation with increase caesarean section rate Int J Reprod Contracept Obstet Gynecol. 2017;6(1):168-71.

9. Dhakare TJ, Patole KP. A study of cardiotocography during active labour to assess the perinatal outcome in high risk pregnancy. MVP J Med Sci. 2016;3(2):92-5.

10. Manning FA, Harman CR, Morrison I, Menticoglou SM, Lange IR, Johnson JM. Fetal assessment based on fetal biophysical profile scoring. IV. An analysis of perinatal morbidity and mortality. Am J Obstet Gynecol. 1990;162(3):703-9.

11. Akhavan S, Lak P, Rahimi-Sharbaf F, Mohammadi SR, Shirazi M. Admission test and pregnancy outcome. Iranian J Med Sci. 2017;42(4):362-8.

12. Hafizur R, Renjhen P, Dutta S, Kar S. Admission cardiotocography: its role in predicting foetal outcome in high-risk obstetric patients. Australas Med J. 2012;5(10):522-7.

13. Kushtagi $P$, Narogoni S. Effectiveness of labour admission test as a screening tool. J Indian Med Assoc. 2002;100(4):234-36. 
14. Ducey J, Guzman E, Schulman H, Farmakedis G, Karmin I. Value of a screening fetal heart rate tracing in the latent phase of labor. J Reprod Med. 1990;35(9):899-900.

15. Ingemarsson I. Electronic foetal monitoring as a screening test. In: Spencer JAD, Ward RHT, eds. Intrapartum foetal surveillance. London: Royal College of Obstetricians and Gynaecologists; 1993:45-52.
Cite this article as: Nandmer GK, Jaiswal S, Namdeo P. Comparative efficacy of admission cardiotocography in high risk and low risk pregnancies in predicting neonatal outcome. Int $\mathbf{J}$ Reprod Contracept Obstet Gynecol 2021;10:3823-7. 